\title{
Segundo cáncer en pacientes pediátricos
}

\author{
M.C. Luisa F. Sepúlveda R. ${ }^{1}$; M.C. Victoría Beresi R. ${ }^{1}$; M.C. Juan Quintana B. ${ }^{1}$; \\ M.C. Humberto del Pozo P. ${ }^{1}$
}

\section{Second malignant tumors in children}

\begin{abstract}
Studies were made on the appearance of second malignant tumors (SMT) in children followed in a pediatric hospital at metropolitan Santiago, Chile, between yeass 1968 and 1987 . A retrospective analysis identified SMT in 7 of 430 patients who survived a childhood cancer (incidence $1.62 \%$ ). An $8^{\text {th }}$ patjent was added, whose first neoplasm was treated in another hospital. The initial diagnosis in the afected children were meduloblastoma, neuroblastoma, Wilm's tumor retinoblastoma, Ewing's arcoma, Hodgkin's disease and, in two cases, acute lymphocytic leukemias. The age range was 6 months to 11 years. Treatment was done by surgery in $5 / 8$, chemotherapy in $7 / 8$ and radiotherapy in all patients. The latent period between the diagnosis of the first cancer and the diagnosis of the SMT was 3.5 to 12 years (median 8.5 years). Osteosarcomas were the most frecuent SMT (5/8). The other SMT were a rhabdomyosarcoma, a non Hodgkin lymphoma and an astrocytoma. The majority of SMT were located in the area of prior radiotherapy $(6 / 8)$. In the other two cases, one had an osteosarcoma, after a bilateral retinoblastoma, which grew outside the previously treated area, and the last one consisted of a lymphoma which was identified 9 years after an acute lymphocytic leukemia. Only 3/8 SMT patients are alive after 14.21 and 34 months follow up. The other children died between 11 and 20 months after diagnosis of SMT. Notwithstanding these kinds of outcome, benefits of therapy for patierts with primary tumors greatly outweight the later risk of cancer induction in a small proportion of them.
\end{abstract}

(Key words: second cancer, radiotherapy, chemotherapy.)

El pronóstico de los pacientes con cáncer infantil ha mejorado considerablemente en los últimos años. En la actualidad es posible la curación de más del $50 \%$ de ellos, debido fundamentalmente a los adelantos en los tratamientos de cirugía, radioterapia y quimioterapia múltiple ${ }^{1-7}$.

Sin embargo, estos tratamientos tienen complicaciones a corto plazo, por efectos tóxicos sobre las células normales del organismo y, a largo plazo, por su potencial acción oncogénica ${ }^{1,3,8,9}$.

Los segundos cánceres (SC) han aumentado debido a mayores sobrevidas y tiempos de seguimiento de los pacientes pediátricos oncológicos. Además, existe cierta predisposición genética a desarrollar más de un cáncer, lo que se ha hecho, tambiên, más manifiesto en las últimas dècadas, como resultado del tratamiento del primer cáncer.

El objetivo de este trabajo es describir los SC observados en nuestro servicio y sus relaciones con el tipo de cáncer inicial y el tratamiento efectuado.

1. Servicio de Oncologia, Hospital Luis Calvo Mackenna

\section{MATERIAL Y PACIENTES}

Se revisó la estadística del servicio de oncología del Hospital Lujs Calvo Mackenna desde 1968 a 1987. En este período fueron atendidos 1.580 pacientes, de los cuales 430 terminaron su tratamiento sin mostrar evidencias de la enfermedad inicial hasta la fecha. En siete de ellos se presentó un SC histológlcamente diferente del primario. Se registraron los detalles de la historia y evolución de cada uno de ellos, y se agregó un octavo caso, cuyo primer tumor fue tratado en otro hospital. Se revisó la distribución por edad, sexo, diagnóstico y tratamientos del cáncer primario, perjodo de latencia en la parición del SC, tratamiento y sobrevida de éste. Finalmente se analizó la relación entre el SC y la radioterapia, observando si el SC se desarrolló dentro o fuera del campo irradiado.

\section{RESULTADOS}

De $\operatorname{los} 1.580$ pacientes tratados en nuestro servicio, 430 terminaron su tratamiento y permanecen sin evidencias de la enfermedad inicial hasta la actualidad. Siete de ellos presentaron un $\mathrm{SC}$, lo que corresponde a una incidencia de $1,62 \%$. En el analisis de los casos se incluye un octavo paciente, cuyo primer cáncer fue trata- 
do en otro hospital. Seis de los 8 pacientes eran de sexo masculino. La edad del conjunto fluctuó entre 6,5 y 20 años, mediana 15,5 años (tabla 1 ).

Los cánceres primarios eran, en un caso cada ило, meduloblastoma, neuroblastoma, tumor de Wilms, retinoblastoma, sarcoma de Ewing, linfoma de Hodgkin y en dos niffos leucemias linfo. blásticas agudas.

El tratamiento efectuado incluyó la extirpa. ción quirúrgica en cinco de los seis tumores sólidos (hizo excepción un paciente con retinoblastoma, por oposición paterna), radioterapia en los ocho pacientes y quimioterapia con varjas drogas en siete (no se hizo esta última en un niño con linfoma de Hodgkin por corresponder al estadio I A) (tabla 1). De los agentes quimioterápicos, la ciclofosfamida se administró en cinco pacientes.

Entre el diagnóstico del primero y segundo cáncer transcurrieron entre 3,5 y 12 años, mediana 8,5 años.

Los SC fueron osteosarcomas en cinco casos, rabdomiosarcoma en uno, astrocitoma maligno en otro y linfoma no Hodgkin en otro más. De los ocho niños que tuvieron SC, sólo viven tres, con periodos de observación entre 14 y 34 meses (tabla 1).

Seis de los ocho SC se localizaron en zona itradiada. El osteosarcoma que apareció fuera del área irradiada ocurrió en un paciente con retinoblastoma bilateral. El otro tumor fue un linfoma
Tabla 2

Segundo cáncer en pacientes pediátricos. Distrỉbución según zona de irradiación

\begin{tabular}{lccc}
\hline $2^{\circ} \mathrm{Ca}$. & $\begin{array}{c}\text { En zona } \\
\text { Irrad. }\end{array}$ & $\begin{array}{c}\text { Fuera zona } \\
\text { Irrad. }\end{array}$ & Total \\
\hline Osteosarcoma & $\mathbf{4}$ & 1 & 5 \\
Rabdomiosarcoma & $\mathbf{1}$ & - & 1 \\
$\begin{array}{l}\text { Astrocitoma } \\
\text { Linfoma }\end{array}$ & 1 & - & 1 \\
\hline & 6 & $\mathbf{1}$ & 1 \\
\hline
\end{tabular}

que apareció nueve ar̃os después a una leucemia linfoblástica aguđa en remisión (tabla 2).

\section{DISCUSION}

La incidencia de SC fue de $1,62 \%$ en esta serie. La incidencia mundial de SC fluctúa entre 1 y $20 \%$ hasta los 20 at̃os de seguimiento en distintas publicaciones ${ }^{1,2,4-6,10,11,13,14,22}$.

La aparición de $\mathrm{SC}$ ha aumentado en las últimas décadas a causa del incremento de la sobrevida de los pacientes oncológicos pediátricos, debida a tratamientos multidisciplinarios más agresivos que incluyen cirugia, radioterapia $y$

Tabla 1

\begin{tabular}{|c|c|c|c|c|c|c|c|c|}
\hline \multicolumn{6}{|c|}{ Ptimier cánier } & \multicolumn{3}{|c|}{ Segundo cánces } \\
\hline \multirow[t]{2}{*}{ Casos } & \multirow{2}{*}{$\begin{array}{c}\text { Sero/edad } \\
\text { diagnóstico } \\
\text { (años) }\end{array}$} & \multirow{2}{*}{$\begin{array}{l}\text { Diagróstico y } \\
\text { localización }\end{array}$} & \multicolumn{3}{|c|}{ Tialamiento } & \multirow{2}{*}{$\begin{array}{r}\text { Lalencia } \\
\text { (meses) }\end{array}$} & \multirow{2}{*}{$\begin{array}{l}\text { Diaznostico y } \\
\text { octulización }\end{array}$} & \multirow{2}{*}{$\begin{array}{c}\text { Scguimiento } \\
\text { (meses) }\end{array}$} \\
\hline & & & Cirugía & Radivterapia & Quimjoterspia & & & \\
\hline 1 & $\mathrm{M} / 11$ & Meduloblasiona & sí & 50 Gy 1umor & Pro: $\forall C R$, MTX & 42 & $\begin{array}{l}\text { Rabdomiosarcoma } \\
\text { masioides det. }\end{array}$ & $14 F$ \\
\hline 2 & $M / 2$ & $\begin{array}{l}\text { Neusoblastoma } \\
\text { supranenal der. }\end{array}$ & si & $30 \mathrm{~Gy}$ thance des. & $C F M, V C R, A D R$ & 52 & $\begin{array}{l}\text { Ustersyjuorna } \\
\text { sactojiliaco des. }\end{array}$ & $20 \mathrm{~F}$ \\
\hline 3 & $F ; B$ & $\begin{array}{c}\text { T. Wilms izq. Il } \\
\text { Metastasss fémur izq. } \\
\text { (3, } 5 \text { ahos después) }\end{array}$ & si & $\begin{array}{l}\text { 48 Gy tose renal izq. } \\
50 \text { Gy fémits izq. }\end{array}$ & $\begin{array}{l}\text { VCR, ACT } \\
\text { CFM, VCR, ADR, ACT }\end{array}$ & 48 & $\begin{array}{l}\text { Osteosarcoma } \\
\text { fémur izq. }\end{array}$ & $14 \mathrm{~F}$ \\
\hline 4 & $\mathrm{M} / \mathrm{S}$ & $\begin{array}{c}\text { Retinoblastoma } \\
\text { bülateral }\end{array}$ & no & 35 Gy ótbitas & CFM, VCR, ADR, ACT & 92 & $\begin{array}{l}\text { Osteosarcoma } \\
\text { tibia izq. }\end{array}$ & $14 \mathrm{~V}$ \\
\hline$s$ & M/11 & T. Kưing peroné s2q. & si & 60 Gy veroné $12 \mathrm{~g}$. & CIM, YCR, PTed, MTX, 6MP & 108 & $\begin{array}{l}\text { Osteosorcoma } \\
\text { tibia izq. }\end{array}$ & $34 \mathrm{~V}$ \\
\hline 6 & $1 / 8$ & $\begin{array}{l}\text { Leucemin linfablästica } \\
\text { aguda }\end{array}$ & no & 24 Gy encéfato & CIM, YCR, ADR. ACNL: & 108 & $\begin{array}{c}\text { Linfoma no Hodekin } \\
\text { anexial }\end{array}$ & $20 \mathrm{Yi}$ \\
\hline 1 & $M / B$ & $\begin{array}{c}\text { Leucrmia Ilin foblástica } \\
\text { aguda }\end{array}$ & no & 20 Gy encéfalo & YCR, ADR, Fed, MTX, GMP & 129 & Astrotitema frontal & $11 \mathrm{H}$ \\
\hline 8 & $M / 3$ & $\begin{array}{l}\text { Linfoma de Hodgkln } \\
\text { cervical iag. } 1 .\end{array}$ & si & 40 Gy manto & & 144 & $\begin{array}{l}\text { Onteosarcoma } \\
\text { húmeso der. }\end{array}$ & $21 \mathrm{~V}$ \\
\hline
\end{tabular}

$\mathbf{I}=$ tumor: I \& I = estzdio I o II: Gy = Grey; Proc = Procarbazina: VCR = Vincristing; MTX = Metotrexato; CFM = Ciclofosfamida; ADR $=$

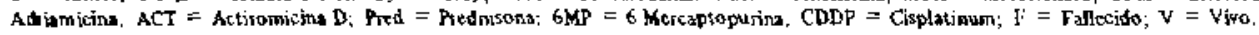


quimioterapia con protocolos más complejos y efectivos.

La larga expectativa de vida de los sobrevivientes de cáncer infantil y la sensibilidad de los niños a los efectos carcinogenéticos de la radioterapia sugieren la posibilidad de un periodo extenso de vida bajo riesgo subsecuente de nuevos cánceres, riesgo que, en estos pacientes, es 10 a 20 veces mayor que en la población general, siendo, al parecer, los factores que más contribuyen a él la predisposición genética y las terapias especificas $1,4,10$.

Se ha encontrado historia familiar de susceptibilidad al cáncer en cerca de $60 \%$ de los pacientes que desarrollaron osteosarcomas como $\mathrm{SC}^{8,24}$. El factor genético ha sido demostrado claramente en los niños con retinoblastoma bilateral, los que tienen alta incidencia de $\mathrm{SC}$; aunque el retinoblastoma es poco frecuente en oncologia pediátrica (aproximadamente $3 \%$ del total) y menos de la mitad son de causa genética, $17 \%$ de los SC ocurrieron entre ellos ${ }^{10}$. El locus para el gen del retinoblastoma está relacionado con la aparición de otros tumores, especialmente osteosarcoma, por lo que éste se presenta a veces en sitios distantes a la irradiación $n^{1,2,4-6,8,10,14}$. En nuestra casuística uno de los SC fue de causa genética (caso 4): tuvo un retinoblastoma bilateral a los 6 meses de edad $y$, 7 1/2 años después, un osteosarcoma de extremidades. Su madre habia sido operada de retino. blastoma unilateral en su infancia $y$, además, hay antecedentes familiares de otros cánceres.

Otras enfermedades genéticas que predisponen al SC son la neurofibromatosis, el xeroderma pigmentoso y el síndrome de carcinoma de célula basal nevoide ${ }^{6,10}$.

La exposición a un carcinógeno conocido precede por muchos años la aparición de un tumor. El período de latencia para el desarrollo del SC es más corto en los casos que tienen predisposición genética y han sido irradiados. Knudson ha sugerido que deben ocurrir dos mutaciones para los cánceres infantiles. La primera puede ser en una célula germinal y la segunda en una célula somática. Para los casos no heredados ambas mutaciones ocurren en células somáticas. En ellos la radioterapia, probablemente, produce la primera mutación y los factores externos la segun$\mathrm{da}^{15}$.

El efecto oncogénico de la radioterapia se debe a que ésta daña al DNA, provocando mutaciones genéticas ${ }^{17,27}$. Ciertos tejidos como el tiroides, la mama y la médula ósea son especialmente radiosensibles. Los SC inducidos por la radioterapia más comunes son los osteosarcomas, las leucemjas y el cáncer tiroideo ${ }^{6,18-21}$. Las leucemias tienen menor tiempo de Jatencia que los tumores sólidos cuando son inducidas por radioterapia $6,14,22$ y su proporción como $\mathrm{SC}$ es menor que la de éstos, lo que se atribuye a que las altas dosis de radioterapia tienden a producjr más bien muerte celular que transformación leucémica $^{1,2,21}$.

Los riesgos de $\mathrm{SC}$ con los equipos actuales de radioterapia, que usan megavoltios, con lo cual se reduce la absorción por piel $y$ huesos, son menores que con los antiguos de ortovolta$\mathrm{je}^{1,2,6,13,21}$. La dosis de radioterapia es directamente proporcional al tiesgo de cáncer óseo ${ }^{10,16}$. En un estudio previo, $75 \%$ de los SC fue asociado a la radioterapias. Todos nuestros pacientes recibieron cobaltoterapia y en $6 / 8$ los $\mathrm{SC}$ ocurrieron en áreas previamente irradiadas, por lo que éstos pueden considerarse secundarios a aquélla.

La acción carcinogenética de la quimioterapia ha sido demostrada en animales de experimentación, siendo más potente en los agentes alquilantes. Estos forman reactantes electrof ílicos por activación espontánea o enzimática y dañan la célula por unión a las mactomoléculas mucleofilicas, incluyendo el $\mathrm{DNA}^{23,25}$. Esta evidencia en el hombre es menos clara. Los agentes especificos más frecuenternente implicados son la ciclofosfamida, el metotrexato y la combinación de procarbazina, prednisona, vincristina y agentes alquilantes. Los menos potentes, en este sentido, son los antimetabolitos ${ }^{22-24}$. De nuestros $8 \mathrm{pa}$ cientes 5 recibieron ciclofosfamida. Sin embargo, la influencia oncogénica de la quimioterapia puede estar obscurecida en ellos, por los muy intensos efectos de la radioterapia asociada.

Cuando se usan conjuntamente radioterapia y quimioterapia los riesgos de $\mathrm{SC}$ son mayores $\mathrm{y}$ el tiempo de latencia es más corto ${ }^{1,11}$. Se ha visto que la quimioterapia agresiva con varios agentes, asociada con grandes dosis de radiación, en el tratamiento del linfoma de Hodgkin, aumenta hasta $20 \%$ en 20 affos el riesgo de leucemia linfoblástica posterior ${ }^{3,4,8,16,18,24,25,27}$. Este sinergismo es aún mayor cuando se usa el esquema MOPP (combinación de mostaza nitrogenada, oncovín, procarbazina y prednisona), en vez del ABVD (adriamicina, bleomicina, vimblastina $y$ dacarbazina) $)^{11,26,27}$. En cambio, la actinomicina 
D junto con la radioterapia parece reducir el riesgo del $\mathrm{SC}^{5,8,25}$.

El SC más frecuente en nuestra serie fue el osteosarcoma, $5 / 8$, lo que concuerda con otras experiencias $^{5,6,10,15}$. Los tipos de tumores de los SC no son, generalmente, los que tienden a ocurrir como cáncer primario en niños. Ningún SC fue tumor embrionario, lo que sugiere que estos son inducidos precozmente, tal vez en el período fetal ${ }^{5,6}$. Los SC más comunes son sarcomas óseos y de tejidos blandos y su instalación en la primera década de la vida, a diferencia de su natural ocurrencia como tumor primario en la segunda o tercera década, pareceria estar influenciada por agentes extemos como la radioterapia $^{5}$. Los SC que se desarrollan después de una leucemia son menos frecuentes que los que siguen a turnores sólidos. Uno de nuestros pacientes (caso 6) tuvo leucemia linfoblástica aguda $y$, tras nueve años de remisión completa, un linfoma no Hodgkin anexial que al poco tiempo sufrió transformación leucémica, por lo cual nos caben dudas si considerarlo como SC o como recaída tardía de la leucemia.

Los tumores inducidos por la radiación no pueden distinguirse de su presentación natural; sin embargo, tienden a comportarse en forma más agresiva y refractaria al tratamiento ${ }^{27}$. Nuestra experiencia fue similar. De los $8 \mathrm{SC}$, 5 fallecieron y los 3 que aún siguen vivos tienen períodos más bien cortos de observación, entre 14 y 34 meses.

Para conocer y evaluar mejor los SC es necesario estudiar grandes poblaciones de pacientes oncológicos tratados y mejorados. Por esta razón, en EE.UU, y Europa se ha formado un grupo cooperativo de doce centros pediátricos de cáncer llamado "The late effects study group"s .

Debemos seguir el control de nuestros pacientes después de la ninez y la adolescencia, alertas a la aparición del SC y otras secuelas del tratamiento. Sin embargo, aun cuando el riesgo del $\mathrm{SC}$ es real los beneficios de las terapias específicas lo sobrepasan con creces. Para reducir la incidencia de SC se está tendiendo en los nuevos protocolos a bajar las dosis de radjoterapia y la intensidad de la quimioterapia en aquellos cán. ceres menos avanzados.

\section{RESUMEN}

Se revisa la casuística de un servicio de oncología pediátrica desde 1968 a 1987. En este periodo se atendieron 1.580 pacientes, 7 de los cuales sufrieron un segurido cáncer ( $\mathrm{SC}$ ). Se incluye un octavo caso, cuyo primer cáncer fue atendido en otro hospital. Los diagnósticos iniciales fueron: meduloblastoma, neuroblastoma, tumor de Wilms, retinoblastoma, sarcoma de Ewing, enfermedad de Hodgkin y dos teucemias linfoblásticas agudas. El tratamiento fue cingía en $5 / 8$, quimioterapia en $7 / 8$ y radioterapia en todos. La latencia entre el primero y el segundo cáncer fue de 3,5 a 12 años (mediana 8,5 años). Los SC fueron osteosarcoma en 5 casos, rabdo miosarcoma en uno, linfoma no Hodgkin en uno y astrocitoma en otro paciente. En $6 / 8$ casos se ubicaron en la zona irradiada. Los otros dos SC fueron un osteosarcoma de extremidades, después de un retinoblastoma bilateral y un linfoma diagnosticado 9 atos después de una leucemia en remisión. Sóla $3 / 8$ pacientes con SC aún sobreviven, cumpliendo entre 14 y 34 meses de observación. Los beneficios de las terapias de los pacientes oncológicos sobrepasan en gran medida al riesgo de un segundo cáncer.

(Palabras claves: segundo cáncer, radiación, quimioterapia.)

\section{REFERENCIAS}

1. Li F.P.: Second Malignant tumors after cancer in chllhood, Cancer 1977;40: 1899-1902.

2. Li F.P., Cassady J.R., Joffe N.: Risk of second tumors in survivors of childhood cancer. Cancer $1975: 35: 1230-1235$

3. Meadow A.: The medical consequence of cure. Cancer 1986: $58: 525$.

4. Byrd R.L.: Late effects of treatment of cancer in children. Pediatric annals $1983 ; 12: 450-460$.

5. Mike V., Meadow A., D'Angio G.: Incidence of Second malignant neoplasms in children. Results of an international study. The Lancet 1982; 2 : 1326-1331.

6. Meadow A.T., D'Angio C.J., Mike V. et al.: Patterns of second malignant neoplasms in children. Cancer 1977; 39: 1903-1911.

7. Sutow W., Vietti $T$. Fernback D.: Capítulos 1, 2 y 3, en: Clinical Pediatric Oncology. 1977:1-42.

8. Giulio f., D'Angio M.D.: Early and delayed complication of therapy. Cancer 1983; $51: 2515-2518$.

9. Byrd R., M.D.: Late effects of treatment of cancer in children. En The Pediatric Clinics of North America. Symposium on Pediatric Oncology. $1985 ; 32: 846-848$.

10. Meadow 4 .: The medical consequence of cure. Cancer $1986 ; 58: 525$.

11. Nelson D.F., Cooper S, Winston M.G. et al. Second malignant neoplasms in pacients treated 
for Hodgkin's disease with radiotherapy or radiotherapy and chemotherapy. Cancer 1981; 48: 2386-2393.

12. Duffner P., Cohen M.: The long term effects of Cranial Irradiation on the S.N.C.. Cancer 1985; 56: 1841-1846.

13. Roger A.: Potish. The incidence of second neoplasms following megavoltage radiation for pediatric tumors. Cancer $1985 ; 56 ; 1534-1537$.

14. Tucker M.A., D'Angio G.J., Bice J.D.: Bone Sarcomas linked to Radiotherapy and Chemotherapy in Children. N Fingl J Med 1987; 317: 588.

15. Meodow A., Strong L.C., Li F.P.: Bone Sarcoma as malignant neoplasm in châldren. Cancer 1980 ; 46: $2600-2603$.

16. Meadow A., D'Angio G., Evans A.E.: Oncogenesis and other late effects of cancer tieatment in children. Radiology $1975 ; 114 ; 135-180$.

17. Mosjiezuk A.D., Roymonn F.B. et al.: Second malignancy in acute lymphocytic leukemia. Am $\mathrm{J}$ Dis Child 1981:135: 313-316.

18. Favis M.J. Schneider A.B., Stachura M.E. et al.: Thyroid cancer ocurring as a late consequence of head and neck irtadiation. N Fngl J Med 1976; 194: 1019-1025.

19. Hutchinson G.B.: Rate neoplastic changes following medical irradiation. Cancer 1976; 37: 1102-3107.
20. Li F.P.: Colon cancer after Wilms tumor. J Pediatric 1980; 96 : 954-955.

21. Heselow R., Neskit M., Dehner P.L.i Second neoplasms following megavoltage radition in a pediatric population. Cancer 1978 ; 42: 11851191.

22. Brody R.S., Schorranfeld D., Reid A.: Multiple primary cancer risk after therapy for Hodgkin disease. Cancer 1977: 40: 1917-1926.

23. Marguardt $H$.: Induction of malignant transformation and mutagenesis in cell cultures by cancer chemotherapeutic agents. Cancer 1977; 40: 1930 1934.

24. Krasse J., Ayuyang H.: Secondary no hematopoietic concer arising following treatment of hematopoietic disorders. Cancer $1985 ; 55$ : 512 515.

25. Harris $C$ : This carcinogenecity of anticancer drugs: a hazard in man. Cancer $1976 ; 37: 1014$ 1023.

26. Bartolucci $A$.: A cute mjelogenous Leukemia as a second malignant nooplasm following the successful treatment of Hodgkin's disease. Cancer 1983; 52: 2209-2213.

27. Cadman E.C., Capizzi R.L., Bertino J.R.: Acute Nonlymphocytic leukemia. A delayed complication of Hodgkin's disease therapy: analisis of 109 cases. Cancer 1977; 40:1280-1296. 